\title{
ENGRAVE: Electromagnetic counterparts of gravitational wave sources at the Very Large Telescope
}

\begin{abstract}
A.J. Levan*
Department of Astrophysics/IMAPP, Radboud University, PO Box 9010, NL-6500 GL Nijmegen, the Netherlands

Email:A.Levan@astro.ru.nl

on behalf of the ENGRAVE collaboration ${ }^{\dagger}$

Email:ec@engrave-eso.org

The "Electromagnetic counterparts of gravitational wave sources at the Very Large Telescope" (ENGRAVE) project is a 200+ person consortium seeking to efficiently utilise the facilities of the European Southern Observatory (ESO) and others to perform in depth observations of electromagnetic counterparts to gravitational wave sources identified in the third observing run (O3) and beyond. In this proceedings we provide a brief overview of the motivation for the formation of such a consortium, a review of the contributions that ESO facilities made to observations of the first event, GW170817, and an outline of the prime scientific goals which we will expect to occupy the consortium, and the community at large, in the coming years.
\end{abstract}

The New Era of Multi-Messenger Astrophysics - Asterics2019

25 - 29 March, 2019

Groningen, The Netherlands

\footnotetext{
* Speaker.

${ }^{\dagger}$ This is a proceedings based on a talk presented at Asterics 2019 by Andrew Levan, a full list of ENGRAVE members can be found at http://www.engrave-eso.org
} 


\section{The Gravitational Wave Electromagnetic Revolution}

The identification of an astrophysical source in both gravitational waves and electromagnetic light has long been awaited. Gravitational waves provide direct information about the motions of masses, enabling the physical mechanisms creating an event to be measured. Combining these with electromagnetic light, which probes the dissipation of energy via various channels, provides a route to building a highly complete model of the physics underlying some of the most extreme events in nature. The strongest expected source of gravitational waves, and the only source-type so far detected, arise from the inspiral of binaries consisting of two compact objects (neutron stars or black holes). Observationally, the most frequent of these events are the binary black hole (BH-BH) mergers, of which 7 were identified during the first two science runs of the LIGO/VIRGO experiment [1]. However, the strength of the measured gravitational wave strain is a strong function of the total system mass, such that BH-BH mergers can be viewed to much larger distances (and hence in much larger volumes) than lower mass systems. This means that the distances to discovered $\mathrm{BH}-$ $\mathrm{BH}$ mergers, typically $\sim 1 \mathrm{Gpc}$, are much larger than the horizons for lighter systems. Combined with the expectation that BH-BH would typically yield weak (or perhaps more likely no) electromagnetic signals, it is unsurprising that despite intensive observational efforts, EM searches for counterparts to BH-BH binaries have not yielded any secured counterpart identifications. Indeed, it is perhaps more surprising that two plausible, though likely unrelated transients - GRB 150914 possibly associated with GW150914 [2] and ATLAS17aeu located in the error box of GW170104 $[3,4]-$ have been located in searches of BH-BH error regions.

Instead, most optimism has been held about the possibility of discovering multiwavelength counterparts of binaries containing neutron stars (either NS-NS or NS-BH). These systems have long been a favoured progenitor for short duration gamma-ray bursts [5] indicating a likely counterpart to an event viewed close enough to its axis. Indeed, the identification in 2013 of a so-called kilonova [6, 7], powered by the synthesis and decay of heavy elements both pointed to a possible isotropic counterpart, and highlighted the role that such binaries may play in the enrichment of the Universe with heavy elements.

\section{GW170817}

The opportunity to study EM emission from a gravitational wave merger arose from GW170817, the first GW event to contain a neutron star, and identified as a NS-NS merger at a distance of approximately $40 \mathrm{Mpc}$. The EM observations have been discussed in detail (see [8,9] and references therein). However, crucially, they enabled the detection of many of the hitherto predicted signatures of a compact binary merger including the detection of a short GRB [10], a kilonova [11, 12, 13, 14] and an off-axis GRB afterglow [15, 16, 17]. Hence, these observations not only demonstrate that EM detections were possible, they suggested that they could be common, and that they could provide much information about the physical mechanisms underlying the mergers.

\subsection{GW170817 observations at the European Southern Observatory}

The facilities of ESO are exceptionally well placed for studies of transient objects thanks to a versatile instrument suite that is normally operated in service mode and hence accessible to 
override observations. Extensive observations of GW170817 were undertaken with ESO facilities. These observations included the independent detection of the source in images taken from the VISTA telescope [14], the first spectrum to show the unusual kilonova features emerging [13], the highest quality polarimetric observations [20], spatially resolved spectroscopy of the host galaxy $[19,18]$, and perhaps most importantly long-lasting spectrophotometric observations of the transient itself $[12,13,14]$,which thanks to the X-shooter spectrograph provided the highest quality spectral sequence available for the merger, with $>10$ epochs, each simultaneously observing from 3000-22000Å. (see Figure 1).

\section{A plan for future observations}

Several features of GW170817 and its follow-up campaign bear comment. Firstly, GW170817 was both unusually close and with a small error box. It lay in the closest $\sim 20 \%$ of the volume accessible to NS-NS mergers during O2, and its error box contained only a few tens of galaxies at an appropriate distance. It was evident that more distant, and more poorly localised events would be more taxing both to locate and follow-up. Secondly, follow-up was intensive but at times redundant, for example similar observations were obtained by multiple (often large aperture) telescopes at comparable times. Thirdly, as the source became fainter the resource investments on large telescopes were such that similar observations could not be made for frequent triggers with the same degree of redundancy.

The ENGRAVE consortium seeks to deliver the highest quality science results with high efficiency through a coherent, well designed, in depth study of the counterparts of gravitational wave sources uncovered during the LIGO/VIRGO O3 run. By combining the science experience of a the majority of the European community and pairing this with the excellent instrumentation available through the facilities of ESO, which at the same time removing duplications and maximising efficiency, enables new science to be effectively undertaken. Our observations will secure data on the next series of gravitational wave counterparts with comparable fidelity to those obtained for GW170817. In particular, our programme has several core aims, including:

- Identify the signatures of nucleosynthesis in the ejecta of compact object mergers and determine their contribution to Universal heavy element enrichment

- Provide redshifts for direct determination of the Hubble constant, and electromagnetic distances to further reduce degeneracies

- Identify afterglows (either off- or on-axis) through deep optical and radio (see below) observations.

- Determine the geometry of kilonova emission through deep (spectro-)polarimetry

- Use integral field spectroscopy (with MUSE) to provide unique constraints on the environments of compact object mergers

ENGRAVE operations are also working beyond the VLT, in particular, there are also components of the programme active at northern sites, such as the $10 \mathrm{~m}$ Grantecan (GTC), as well as 


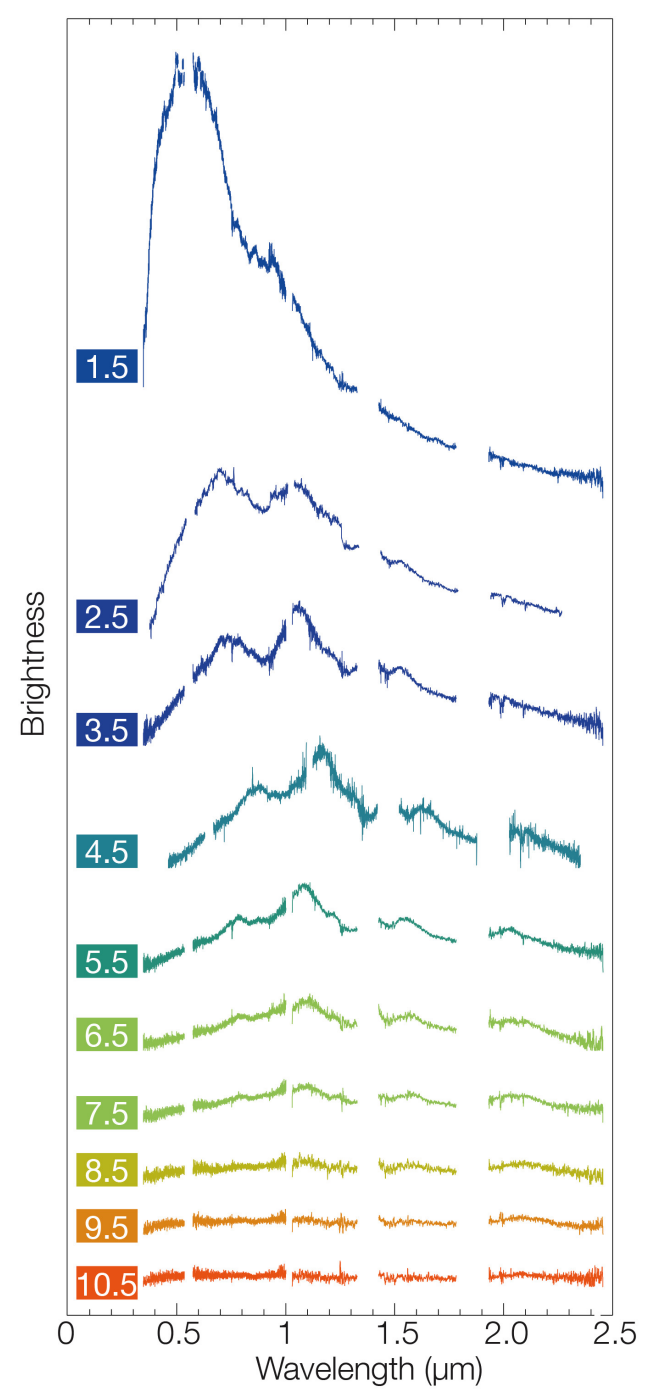

Figure 1: The spectroscopic sequence of observations of GW170817 obtained with the X-shooter instrument at the VLT $([12,13])$. The observations provide daily spectroscopic information across the region of the visible/IR spectrum observable from the ground. ENGRAVE observations will provide similar spectroscopic sequences for future events (image credit: E. Pian, S. Smartt and the European Southern Observatory) 
with the Hubble Space Telescope (HST). There is also a significant longer wavelength component, focussed substantially on the Atacama Large Milimeter Array (ALMA, e.g. [21]), but encompassing other major observatories such as the Jansky Very Large. Array (JVLA), the Giant Metrewave Radio Telescope (GMRT) and the Australian Telescope Compact Array (ATCA) telescopes.

ENGRAVE is focussed on the follow-up of counterparts, and not their identification. However, ENGRAVE members are also heavily involved in identification efforts from a range of facilities across the world, providing start-to-finish expertise within the consortium. These efforts include some with ESO own facilities including searches run from the VLT survey telescope and VISTA.

Ultimately, these observations should, with high efficiency, map out the multiwavelength properties of a modest sample of electromagnetic counterparts to GW sources and begin to answer many of the central questions in contemporary astrophysics which are accessible through such data.

\section{References}

[1] The LIGO Scientific Collaboration, the Virgo Collaboration, Abbott, B. P., et al. 2018, arXiv e-prints, arXiv:1811.12907

[2] Connaughton, V., Burns, E., Goldstein, A., et al. 2016, Astrophysical Journal, 826, L6

[3] Stalder, B., Tonry, J., Smartt, S. J., et al. 2017, Astrophysical Journal, 850, 149

[4] Melandri, A., Rossi, A., Benetti, S., et al. 2019, Astronomy \& Astrophysics, 621, A81

[5] Gehrels, N., Sarazin, C. L., O’Brien, P. T., et al. 2005, Nature, 437, 851

[6] Tanvir, N. R., Levan, A. J., Fruchter, A. S., et al. 2013, Nature, 500, 547

[7] Berger, E., Fong, W., \& Chornock, R. 2013, Astrophysical Journal, 774, L23

[8] Abbott, B. P., Abbott, R., Abbott, T. D., et al. 2017, Astrophysical Journal, 848, L12

[9] Coulter, D. A., Foley, R. J., Kilpatrick, C. D., et al. 2017, Science, 358, 1556

[10] Abbott, B. P., Abbott, R., Abbott, T. D., et al. 2017, Astrophysical Journal, 848, L13

[11] Arcavi, I., Hosseinzadeh, G., Howell, D. A., et al. 2017, Nature, 551, 64

[12] Pian, E., D'Avanzo, P., Benetti, S., et al. 2017, Nature, 551, 67

[13] Smartt, S. J., Chen, T.-W., Jerkstrand, A., et al. 2017, Nature, 551, 75

[14] Tanvir, N. R., Levan, A. J., González-Fernández, C., et al. 2017, Astrophysical Journal, 848, L27

[15] Troja, E., Piro, L., van Eerten, H., et al. 2017, Nature, 551, 71

[16] D’Avanzo, P., Campana, S., Salafia, O. S., et al. 2018, Astronomy \& Astrophysics, 613, L1

[17] Lyman, J. D., Lamb, G. P., Levan, A. J., et al. 2018, Nature Astronomy, 2, 751

[18] Hjorth, J., Levan, A. J., Tanvir, N. R., et al. 2017, Astrophysical Journal, 848, L31

[19] Levan, A. J., Lyman, J. D., Tanvir, N. R., et al. 2017, Astrophysical Journal, 848, L28

[20] Covino, S., Wiersema, K., Fan, Y. Z., et al. 2017, Nature Astronomy, 1, 791

[21] Kim, S., Schulze, S., Resmi, L., et al. 2017, Astrophysical Journal, 850, L21 